\title{
Biomechanical Effects of Tibial Plateau Levelling Osteotomy on Joint Instability in Normal Canine Stifles: An In Vitro Study
}

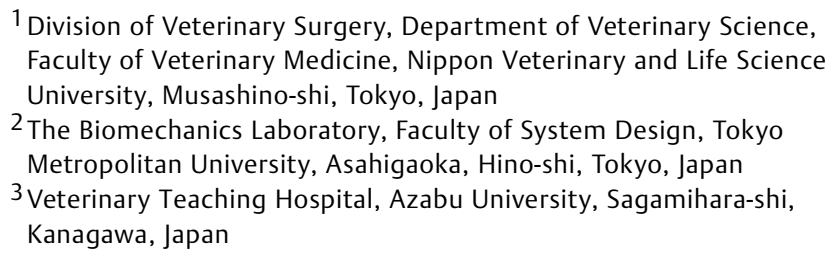

Masakazu Shimada ${ }^{1}$ Tetsuya Takagi ${ }^{2}$ Nobuo Kanno ${ }^{1}$ Satoshi Yamakawa ${ }^{2}$ Hiromichi Fujie ${ }^{2}$ Tom Ichinohe ${ }^{1,3}$ Shuji Suzuki ${ }^{1}$ Yasuji Harada ${ }^{1}$ Yasushi Hara ${ }^{1}$

Address for correspondence Masakazu Shimada, DVM, Division of Veterinary Surgery, Department of Veterinary Science, Faculty of Veterinary Medicine, Nippon Veterinary and Life Science University, 1-7-1 Kyonan-cho, Musashino-shi, Tokyo 180-8602, Japan (e-mail: masakazu42710@gmail.com).

Vet Comp Orthop Traumatol 2020;33:301-307.

\begin{abstract}
Objective The aim of the study was to determine the changes in biomechanical characteristics following tibial plateau levelling osteotomy (TPLO) using simulated manual tests.

Study Design Twenty-one stifles from healthy Beagle dogs that had undergone TPLO or had not (control) were first tested in the intact form, and then the cranial cruciate ligament $(\mathrm{CrCL})$ was transected in each to provide four test situations: control-intact, control-CrCL-transected, TPLO-intact and TPLO-CrCL-transected. The stifles were then analysed using a robotic joint biomechanical testing system. The craniocaudal drawer, axial rotation and proximal compression tests were applied.

Results The craniocaudal displacement during the drawer test was not significantly

Keywords

- dog

- biomechanical effect

- six-degree-offreedom robotic testing system

- cranial cruciate ligament

- tibial plateau levelling osteotomy different between the control-intact and TPLO-intact. However, the displacement was significantly greater in the TPLO-CrCL-transected than in the control-intact. In the axial rotation test, the internal-external (IE) rotation was significantly greater in the TPLOintact than in the control-intact. Similarly, the IE rotation was significantly greater in the TPLO-CrCL-transected than in the control-CrCL-transected. In the proximal compression test, craniocaudal displacement was not significantly different among the controlintact, TPLO-intact and TPLO-CrCL-transected.

Conclusion These findings suggest that TPLO influences the tension of the collateral ligaments and might generate laxity of the tibiofemoral joint. Instability after the osteotomy might be associated with the progression of osteoarthritis.
\end{abstract}

\section{Introduction}

Cranial cruciate ligament $(\mathrm{CrCL})$ rupture is an orthopaedic disease that occurs with high frequency in dogs. Dynamic stabilizers, such as the quadriceps muscle and static stabil- izers, such as the joint capsule, ligaments and meniscus, stabilize the stifle joint. The major ligaments that mediate stifle stabilization are the $\mathrm{CrCL}$, caudal cruciate ligament, medial collateral ligament and lateral collateral ligament. The CrCL plays a vital role in maintaining stifle stability by received

April 15, 2019

accepted after revision

February 24, 2020

published online

April 13, 2020 (c) 2020 Georg Thieme Verlag KG Stuttgart · New York
DOI https://doi.org/ $10.1055 / \mathrm{s}-0040-1709505$. ISSN 0932-0814. 
preventing cranial tibial drawer movement, limiting joint hyperextension and preventing excessive internal tibial rotation, in combination with the caudal cruciate ligament. ${ }^{1}$

The clinical efficacy of tibial plateau levelling osteotomy (TPLO) is widely recognized. Tibial plateau levelling osteotomy is a functional stabilization method that was first described by Slocum and Slocum. ${ }^{2}$ This surgical method involves an osteotomy and rotation of the proximal tibia to correct the tibial plateau angle (TPA). The goal of TPLO is to neutralize cranial tibial thrust and prevent cranial displacement of the tibia in the stance phase. ${ }^{2}$

Tibial plateau levelling osteotomy is a useful treatment for $\mathrm{CrCL}$ rupture because it provides early functional restoration. ${ }^{3}$ However, TPLO cannot prevent the progression of osteoarthritis secondary to CrCL rupture. ${ }^{4,5}$ Osteoarthritis may develop due to chronic ligament degeneration, greater stifle instability following CrCL rupture and changes in biomechanical characteristics following the TPLO-induced changes in the anatomical conformation of the tibia. Previous studies have compared stifles that have undergone $\mathrm{CrCL}$ resection and TPLO with intact joints. ${ }^{6-8}$ However, in such studies, it is difficult to attribute changes in kinematics specifically to either CrCL resection or TPLO. Therefore, we aimed in the present study to determine not only the effect of CrCL resection, but also the changes induced by TPLO, by comparing the effects of the presence and absence of $\mathrm{CrCL}$ in control and TPLO-treated stifles. Many previous studies have shown changes in stability induced by axial loading or during the craniocaudal drawer test. ${ }^{6-9}$ However, the $\mathrm{CrCL}$ is also considered to play a role in the resistance of the joint to axial rotational force; therefore, we also aimed to assess the effects of TPLO and CrCL on this parameter.

We hypothesized that TPLO stabilizes the joint with respect to axial loading, but creates instability with respect to cranial drawer and axial rotation. Therefore, we aimed to determine the biomechanical effects of TPLO on stifle joint stability, by performing the craniocaudal drawer, axial rotation and proximal compression tests in control and TPLOtreated stifles, with and without an intact cranial cruciate ligament, using a robotic system.

\section{Materials and Methods}

\section{Animals}

The stifle joints used in this study were obtained from 21 healthy Beagle dogs that had been euthanatized for other reasons. The stifles were divided into two groups: those that did not undergo TPLO (control group) and those that did (TPLO group). The characteristics of each group are shown in - Table 1. This study was approved by the Animal Experiment Committee and Bioethics Committee of our university (approval number: 28S-57).

\section{Specimen Preparation}

All soft tissues, except the collateral ligaments, the cruciate ligaments, menisci, joint capsule, patella and patellar ligament, were removed from the stifle joint, to create a bone-ligament model, based on the method of Kanno and colleagues. ${ }^{9} \mathrm{~A}$
Table 1 Characteristics of the control and TPLO groups

\begin{tabular}{|l|l|l|}
\hline Group & Control & TPLO \\
\hline Number & $n=10$ & $n=11$ \\
\hline Age (months) & $21.4 \pm 5.9$ & $14.9 \pm 3.1$ \\
\hline Sex, M:F & $9: 1$ & $6: 5$ \\
\hline Body weight $(\mathrm{kg})$ & $11.2 \pm 1.3$ & $11.2 \pm 1.9$ \\
\hline
\end{tabular}

Abbreviations: F, female; M, male; TPLO, tibial plateau levelling osteotomy. Note: Data are presented as mean \pm standard deviation.

mediolateral radiograph of each stifle was obtained, and the TPA was measured according to the method of Warzee and colleagues. ${ }^{6} \mathrm{~A}$ craniocaudal radiograph was also obtained to check for deformities, such as varus or valgus. In the TPLO group, surgery was performed according to the method of Slocum and Slocum. ${ }^{2}$ We performed an osteotomy, rotated the proximal segment and applied temporary fixation using Kirschner wire. Radiographs were then obtained and the TPA was measured again. If the TPA was within the range of $6.5 \pm 2.0$ degrees, we performed fixation using a $2.4-\mathrm{mm}$ TPLO locking compression plate (Johnson \& Johnson; New Brunswick, New Jersey, United States). After processing, radiographs were again obtained and the postoperative TPA was measured. Next, the proximal femur and distal tibia were fixed using dental resin (GC OSTRON II; GC Corporation, Tokyo, Japan) and a cylindrical paper tube. To standardize between specimens, Kirschner wires were inserted in the bone axis of the femur and tibia. The resin was fixed at the centre of the tube so that the wires could be clamped by the robot. After fixing the bones with screws from all sides, so that the Kirschner wires were perpendicular to the centre of the tube, the resin was placed into the tube. At this time, the insertions of the medial and lateral collateral ligaments were marked with a surgical pen, because the coordinate system was determined on the basis of these marks. The specimens were then wrapped with gauze soaked in lactated Ringer's solution and cryopreserved at $-20^{\circ} \mathrm{C}$. They were thawed at $4^{\circ} \mathrm{C}$ the day before examination and testing by the robotic system.

\section{Six-Degree-of-Freedom Robotic Testing System}

A custom-made six-degree-of-freedom robotic testing system developed by Fujie and colleagues ${ }^{10-13}$ was used for the testing (-Fig. 1). This system enables the simulation of physiological stifle joint motion that is controlled with respect to either position or force, using a coordinate system (Grood and Suntay $^{14}$ ) in vitro. ${ }^{12}$ The coordinate system consists of three axes that are used to assess rotation and translation in six-degree-offreedom. The motion can be defined in terms of three rotations (flexion-extension, internal-external [IE], varus-valgus) and three translations (medial-lateral, craniocaudal, proximal-distal)( - Fig. 2). In the robotic system, the flexion-extension axis is defined using the femur and the IE rotation axis is defined using the tibia based on bone landmarks and the manipulator position. The varus-valgus axis is defined by the line perpendicular to the flexion-extension and IE rotation axes. This new robotic system utilizes a real-time controller, which made it possible to speed up the control cycle in this study. ${ }^{10}$ 


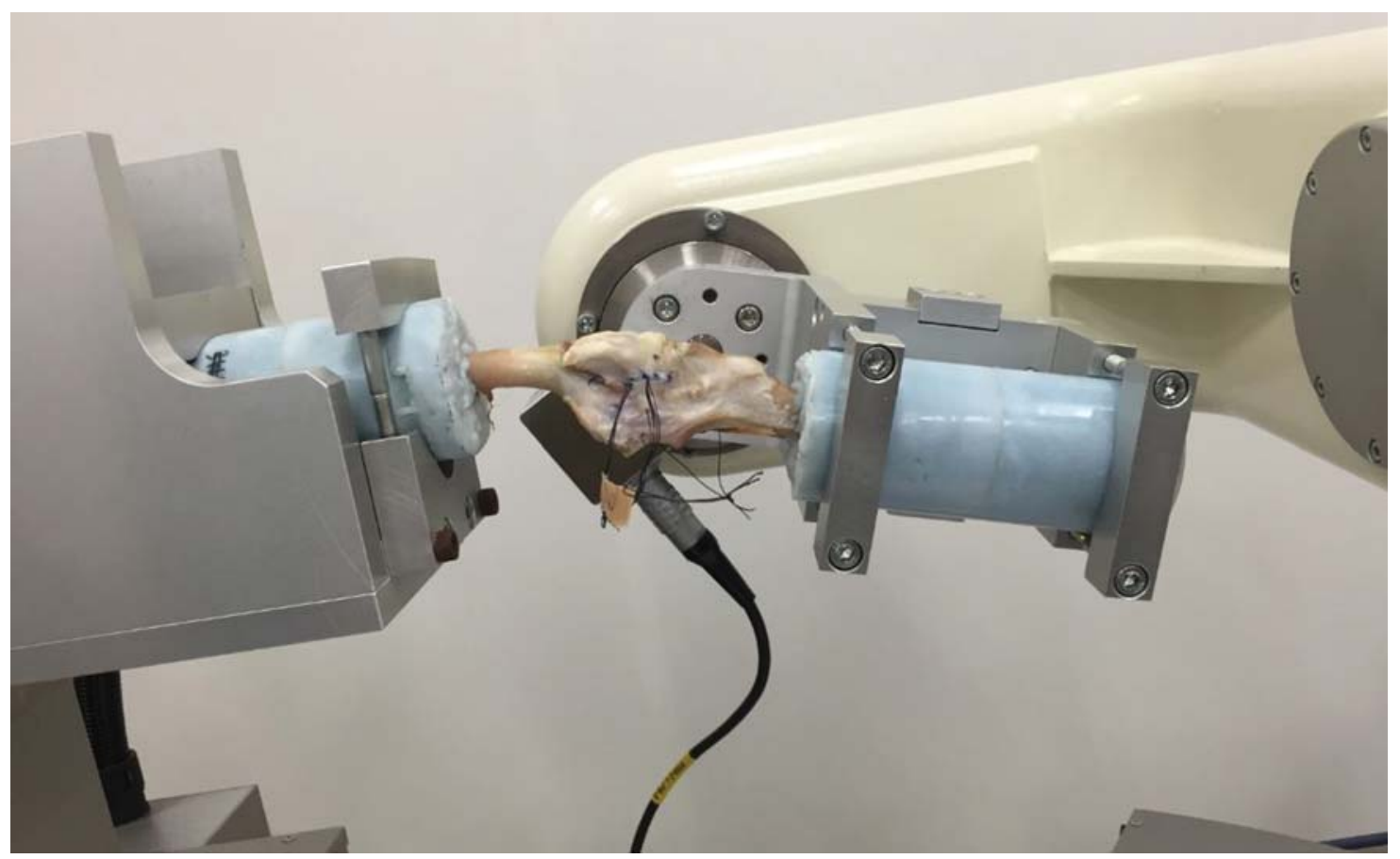

Fig. 1 The testing system used in this study. This consisted of a 6-degree-of-freedom manipulator with a 6-degree-of-freedom universal force/moment sensor. The femur is on the left and the tibia is on the right. The suture coming out of the joint is placed as a mark when the cranial cruciate ligament is cut. The cable transmits the data from the sensor to the computer.

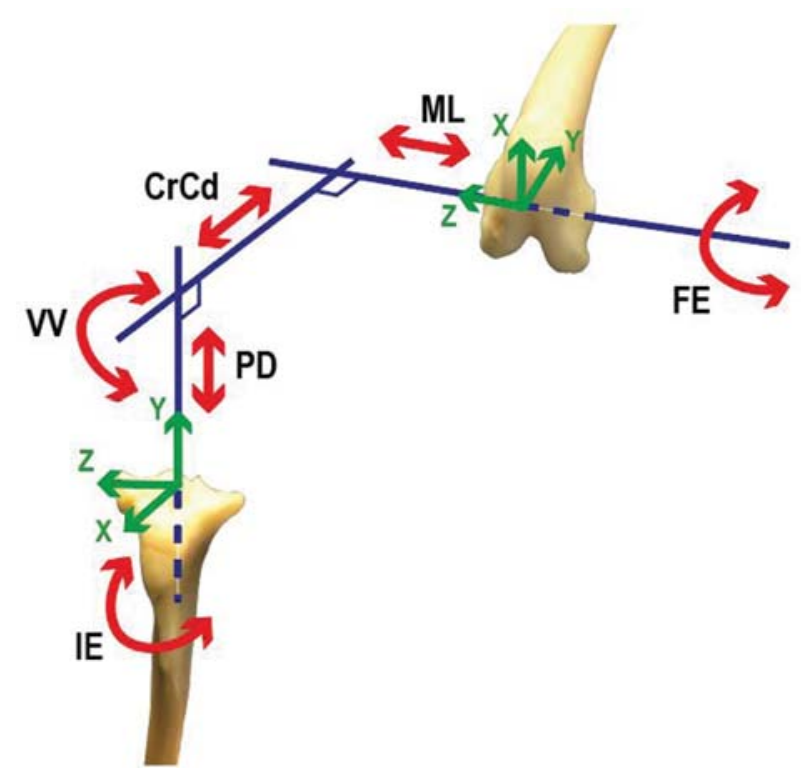

Fig. 2 The stifle coordinate system and stability control in each direction. This coordinate system consists of three rotational axes and their translations: flexion-extension (FE), internal-external (IE), varus-valgus (VV), medial-lateral (ML), cranial-caudal ( $\mathrm{CrCd}$ ) and proximal-distal (PD).

\section{Testing Protocol}

The stifle was extended as much as possible, with $0.5 \mathrm{Nm}$ of extension torque, and the flexion-extension angle of the stifle, measured using a goniometer, was defined as the extension position. The mean extension of the control group was 153 degrees; therefore, 153 degrees was used as the extension value for the TPLO group. The craniocaudal drawer, axial rotation and proximal compression tests were performed at this degree of extension, and at 135 and 120 degrees, using the robotic testing system. In the craniocaudal drawer test, as for the cranial drawer test, ${ }^{15}$ craniocaudal drawer loads of up to $30 \mathrm{~N}$ were applied to the stifles, while maintaining the joint angle and keeping the other four-degree-of-freedom forces/ torques at 0 . The six-degree-of-freedom displacement was then recorded and the range of motion during the craniocaudal displacement was calculated. In the axial rotation test, $1 \mathrm{Nm}$ of IE torque was applied to the stifle joints, as in the craniocaudal drawer test, to measure the range of motion during IE rotation. In the proximal compression test, to mimic the method of clinical diagnosis (the positive cranial tibial thrust test), ${ }^{15} 30 \mathrm{~N}$ of proximal force was applied to the stifle joint, while maintaining all the rotation angles and keeping the medial-lateral and craniocaudal forces at 0 ; the six-degree-of-freedom displacement was then recorded. This test was used to assess craniocaudal displacement.

\section{Test Situations}

The stifles were allocated to TPLO and control groups, tested as intact stifles and then the $\mathrm{CrCL}$ were transected in half the joints (CrCL-transected) to provide four test situations: control-intact, control-CrCL-transected, TPLO-intact and TPLO-CrCL-transected.

\section{Statistical Analysis}

SPSS software version 26 (SPSS Inc., Chicago, Illinois, United States) was used for the statistical analysis. One-way analysis of variance was performed in each case, then to compare the changes between each tested joint angle, Tukey's honest significant difference test was used post hoc. Comparisons among the groups were made using the Tukey-Kramer test. Differences were considered to be significant when $p<0.05$. 


\section{Results}

The TPA in the control group was $32.0 \pm 3.0$ degrees. In the TPLO group, the preoperative TPA was $30.4 \pm 2.6$ degrees and the postoperative TPA was $6.4 \pm 2.6$ degrees. The displacement values for each test are shown in -Tables $\mathbf{2}$ and $\mathbf{3}$.

\section{Comparisons of Differences in the Tested Joint Angles}

\section{Craniocaudal Drawer Test}

No significant differences in the tested angles were found in the control-intact and TPLO-intact groups, but in the controlCrCL-transected and TPLO-CrCL-transected groups the craniocaudal displacement was larger at both 135 and 120 degrees than at extension. Therefore, following CrCL transection, there was a tendency for the value to increase with flexion.

\section{Axial Rotation Test}

In the control-intact and TPLO-intact groups, significantly greater IE rotation was found at 135 and 120 degrees than at extension, and at 120 than at 135 degrees. In the controlCrCL-transected and TPLO-CrCL-transected groups, there was greater IE rotation at both 135 and 120 degrees than at extension. Therefore, in all these situations, there was a tendency for the value to increase with flexion.

\section{Proximal Compression Test}

In the control-intact and control-CrCL-transected groups, there was significantly greater craniocaudal displacement at both 135 and 120 degrees than during extension. In TPLOintact and TPLO-CrCL-transected groups, no significant differences in the tested angles were found. Therefore, following $\mathrm{CrCL}$ transection, there was a tendency for the value to increase with flexion.

\section{Comparisons among the Joint Situations}

\section{Craniocaudal Drawer Test}

At each angle, the craniocaudal drawer test result showed significantly greater craniocaudal displacement in TPLOCrCL-transected stifles than in control-intact, controlCrCL-transected or TPLO-intact stifles. In addition, controlCrCL-transected stifles showed significantly greater craniocaudal displacement than control-intact stifles. However, no significant difference was found between control-intact and TPLO-intact stifles. Therefore, there was a tendency for the value to increase following $\mathrm{CrCL}$ transection.

\section{Axial Rotation Test}

In extension, there was significantly less IE rotation in controlintact than in control-CrCL-transected, TPLO-intact or TPLOCrCL-transected stifles. In addition, there was significantly greater IE rotation in TPLO-CrCL-transected stifles than in control-CrCL-transected and TPLO-intact stifles. At 135 degrees, there was significantly less IE rotation in control-intact than in TPLO-intact or TPLO-CrCL-transected stifles. In addition, there was significantly greater IE rotation in TPLOCrCL-transected stifles than in control-CrCL-transected stifles.

Table 2 Comparisons of differences in the outcome measures between the tested joint angles

\begin{tabular}{|c|c|c|c|}
\hline & \multicolumn{3}{|c|}{ Craniocaudal displacement at craniocaudal drawer test (mm) } \\
\hline & Extension & $135^{\circ}$ & $120^{\circ}$ \\
\hline Control-intact & $1.75 \pm 0.48$ & $1.95 \pm 0.67$ & $1.75 \pm 0.77$ \\
\hline Control-CrCL-transected & $4.33 \pm 2.09^{a, b}$ & $6.16 \pm 2.91^{a}$ & $6.93 \pm 3.35^{b}$ \\
\hline TPLO-intact & $2.91 \pm 0.72$ & $2.85 \pm 0.97$ & $2.81 \pm 0.87$ \\
\hline \multirow{3}{*}{$\begin{array}{l}\text { TPLO-CrCL- } \\
\text { transected }\end{array}$} & $10.37 \pm 3.58^{a, b}$ & $11.37 \pm 3.63^{\mathrm{a}}$ & $12.17 \pm 3.84^{b}$ \\
\hline & \multicolumn{3}{|c|}{ Internal-external rotation at axial rotation test $\left({ }^{\circ}\right)$} \\
\hline & Extension & 135 degrees & 120 degrees \\
\hline Control-intact & $44.75 \pm 7.56^{\mathrm{a}, \mathrm{b}}$ & $65.65 \pm 9.10^{\mathrm{a}, \mathrm{c}}$ & $74.45 \pm 7.84^{\mathrm{b}, \mathrm{c}}$ \\
\hline Control-CrCL-transected & $61.77 \pm 6.17^{\mathrm{a}, \mathrm{b}}$ & $75.22 \pm 7.39^{a}$ & $78.05 \pm 9.13^{b}$ \\
\hline TPLO-intact & $72.29 \pm 8.26^{\mathrm{a}, \mathrm{b}}$ & $84.50 \pm 8.64^{\mathrm{a}, \mathrm{c}}$ & $89.45 \pm 8.02^{b, c}$ \\
\hline \multirow{2}{*}{ TPLO-CrCL-transected } & \multicolumn{3}{|c|}{ Craniocaudal displacement at proximal compression test (mm) } \\
\hline & Extension & 135 degrees & 120 degrees \\
\hline Control-intact & $0.14 \pm 0.05^{\mathrm{a}, \mathrm{b}}$ & $0.32 \pm 0.23^{a}$ & $0.30 \pm 0.14^{b}$ \\
\hline Control-CrCL-transected & $1.40 \pm 1.04^{\mathrm{a}, \mathrm{b}}$ & $3.55 \pm 1.84^{a}$ & $3.80 \pm 2.24^{b}$ \\
\hline TPLO-intact & $1.13 \pm 0.10$ & $0.12 \pm 0.09$ & $0.10 \pm 0.07$ \\
\hline TPLO-CrCL-transected & $0.49 \pm 0.70$ & $1.00 \pm 1.33$ & $1.06 \pm 1.27$ \\
\hline
\end{tabular}

Abbreviations: $\mathrm{CrCL}$, cranial cruciate ligament; TPLO, tibial plateau levelling osteotomy. Note: Data are presented as mean \pm standard deviation.

${ }^{a} p<0.05$, extension vs. 135 degrees.

${ }^{\mathrm{b}} \mathrm{p}<0.05$, extension vs. 120 degrees.

${ }^{c} p<0.05,135$ vs. 120 degrees. 
Table 3 Comparisons of differences in the outcome measures among the stifle situations

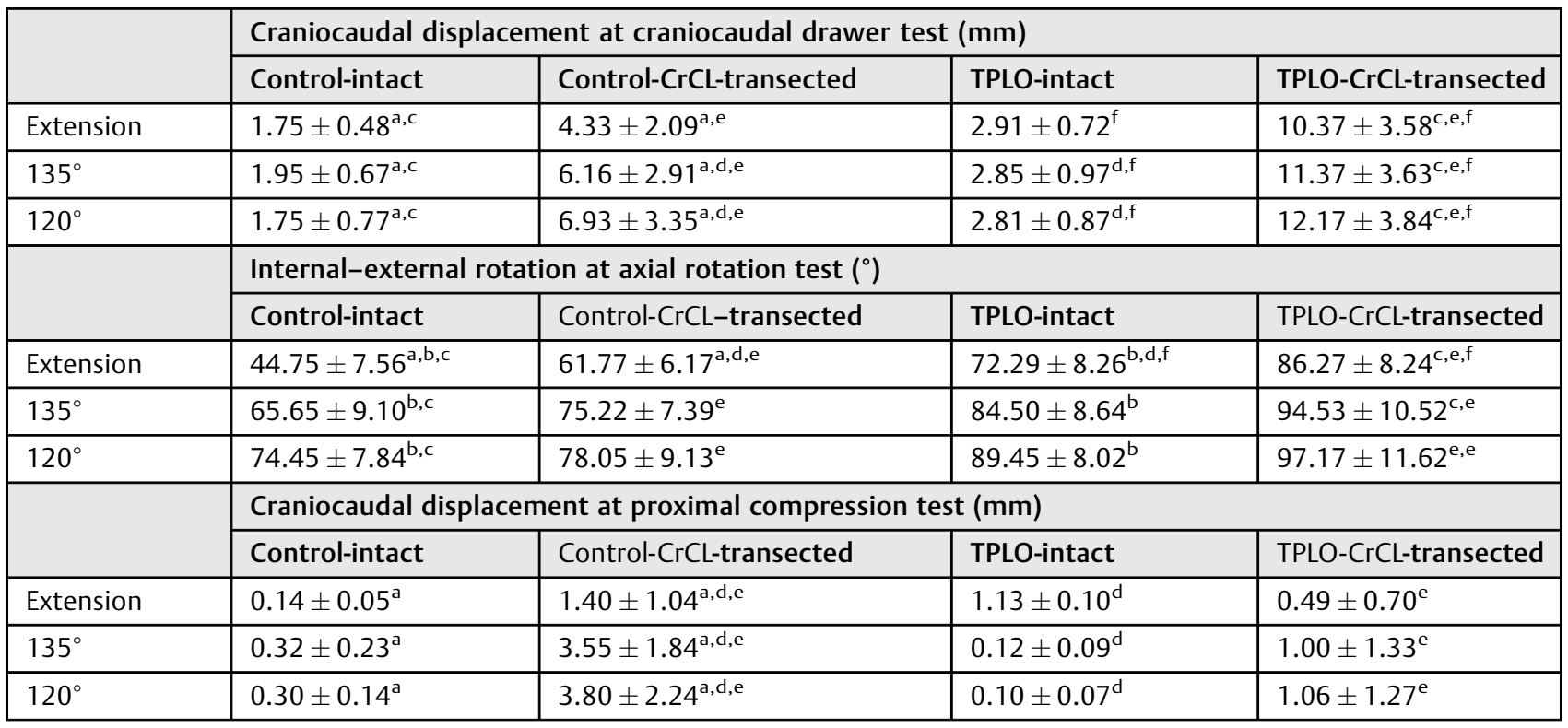

Abbreviations: CrCL, cranial cruciate ligament; TPLO, tibial plateau levelling osteotomy.

Note: Data are presented as mean \pm standard deviation.

${ }^{a} p<0.05$, control-intact vs. control-CrCL-transected.

${ }^{\mathrm{b}} p<0.05$, control-intact vs. TPLO-intact.

${ }^{c} p<0.05$, control-intact vs. TPLO-CrCL-transected.

${ }^{\mathrm{d}} p<0.05$, control-CrCL-transected vs. TPLO-intact.

${ }^{\mathrm{e}} p<0.05$, control-CrCL-transected vs. TPLO-CrCL-transected.

${ }^{\mathrm{f}} p<0.05$, TPLO-intact vs. TPLO-CrCL-transected.

However, there was no significant difference between controlCrCL-transected and TPLO-CrCL-transected stifles or between TPLO-intact and TPLO-CrCL-transected stifles. At 120 degrees, there was significantly less IE rotation in control-intact stifles than in TPLO-intact or TPLO-CrCL-transected stifles. However, there was no significant difference between control-CrCLtransected and TPLO-CrCL-transected stifles or between TPLO-intact and TPLO-CrCL-transected stifles. Therefore, there was a tendency for the value to increase following CrCL transection or with TPLO.

\section{Proximal Compression Test}

At each angle, there was significantly greater craniocaudal displacement in control-CrCL-transected than in controlintact, TPLO-intact or TPLO-CrCL-transected stifles during the proximal compression test. However, no significant differences were found between control-intact and TPLOintact, control-intact and TPLO-CrCL-transected and TPLOintact and TPLO-CrCL-transected stifles. Therefore, there was a tendency for the value to increase following $\mathrm{CrCL}$ transection in the control stifles and to decrease following TPLO.

\section{Discussion}

In this study, we found that TPLO provided stability during craniocaudal movement when a compressive force was applied to the tibiofemoral joint. However, in the absence of a compressive force, we found that TPLO promoted instability in the craniocaudal movement and IE rotation.
The craniocaudal drawer test confirmed that the $\mathrm{CrCL}$ prevents craniocaudal displacement, as previously shown. ${ }^{1}$ Additionally, although no significant differences in craniocaudal displacement were found between the tested joint angles in control-intact stifles, the amount of displacement increased with flexion in control-CrCL-transected stifles. This may be because the collateral ligaments stretch during extension ${ }^{16}$ and stability in the craniocaudal direction may be minimally affected by relaxation of the collateral ligaments during flexion, because the main craniocaudal stabilizer is the $\mathrm{CrCL}$. However, after $\mathrm{CrCL}$ transection, the craniocaudal stabilizing function of the $\mathrm{CrCL}$ is lost, and the collateral ligaments may compensate for some of its functions. Tibial plateau levelling osteotomy promotes greater instability during the craniocaudal drawer test in stifles lacking a CrCL. Thus, TPLO increases flexion at the femorotibial joint, which promotes conformational changes by effects on the collateral ligaments and stifle joint stability. One specific factor may be caudal movement of the insertion on the tibial side, due to rotation of part of the proximal tibia (-Fig. 3). Therefore, TPLO is likely to increase the impact of the absence of a CrCL on joint function.

The axial rotation test in the control group showed that the $\mathrm{CrCL}$ limits IE rotation, so that rotational laxity was more limited during extension than during flexion in the normal stifles. The joint is stabilized against internal rotation by the $\mathrm{CrCL}$ and caudal cruciate ligament, and these ligaments stretch as the joint extends. ${ }^{1}$ However, the collateral ligaments also play a role in limiting axial rotation and stretching 


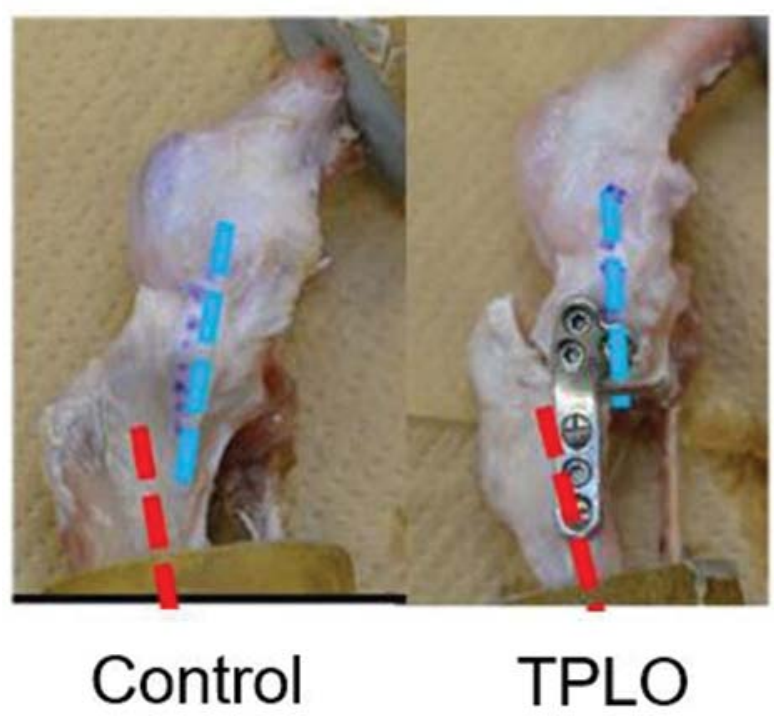

Fig. 3 Stifles in the control and tibial plateau levelling osteotomy groups. The red line represents the anatomical axis of the tibia and the blue line represents the path of the medial collateral ligament. The tibial plateau levelling osteotomy-treated stifle has a more caudal path than the Control stifle.

during extension. ${ }^{16}$ The stretching of each ligament stabilizes the joint against internal rotation in the extended position; however, in the flexed position these ligaments relax and their stabilizing effect is lower. This suggests that the degree of rotational instability depends on the angle of the stifle joint, and our findings suggest that TPLO promotes instability with respect to IE rotation. We think that this may be related to the effect of TPLO on each ligament, as discussed above, and that this instability may explain the sporadic occurrence of the pivot-shift phenomenon postoperatively in clinical cases. ${ }^{17}$

The results of the proximal compression test in the control group suggest that the $\mathrm{CrCL}$ prevents craniocaudal movement in response to a compressive force. When a vertical compressive force is generated at the tibiofemoral joint, as during weight bearing, cranial tibial thrust is generated, which displaces the tibia forward. ${ }^{18}$ Our findings are consistent with those of a previous study of $\mathrm{CrCL}$ function, which showed that the ligament prevents cranial displacement and excessive internal rotation of the tibia. ${ }^{1}$ Grood and Suntay ${ }^{14}$ used a similar six-degree-of-freedom robotic testing system and reported instability in the normal canine stifle joint, identified using the craniocaudal drawer and proximal compression tests in the normal stifles of Beagles. Their finding was similar to ours in that the $\mathrm{CrCL}$ resisted craniocaudal displacement in the presence or absence of a compressive force at the tibiofemoral joint. ${ }^{14}$ We were able to reconfirm these functions of the $\mathrm{CrCL}$ using our robotic system. Furthermore, stifles from TPLOtreated dogs without a $\mathrm{CrCL}$ resisted cranial displacement of the tibia with a compressive force equal to that of normal stifle joints.

Tibial plateau levelling osteotomy promotes axial rotational and craniocaudal instability following $\mathrm{CrCL}$ transection, which may be due to the relative joint flexion created, which induces collateral ligament laxity. However, normal stifles also have static and dynamic stabilizers, such as the quadriceps and hamstring muscles. The quadriceps muscle has a particularly potent influence on the stifle. During extension of the femorotibial joint, the patella creates a retropatellar force that pushes the distal femur caudally. ${ }^{19}$ Through this retropatellar force and cranial tibial thrust, the tibia is displaced cranially, especially in the stance phase. ${ }^{18,19}$ The present study and many in vitro studies, including that of Warzee and colleagues, ${ }^{6}$ have shown an increase in rotational instability in the absence of the $\mathrm{CrCL}$; however, the dynamic stabilizers could not be completely reproduced in these studies. ${ }^{6,9}$ Tashman and colleagues ${ }^{20}$ monitored the kinematics of the stifle joint in foxhounds for 2 years after CrCL excision. Two months after $\mathrm{CrCL}$ excision, the authors noted tibial subluxation in the stance phase and caudal reduction of the tibia in the swing phase. ${ }^{20}$ They also found greater displacement in the craniocaudal direction $(10 \mathrm{~mm}){ }^{20}$ Two years after $\mathrm{CrCL}$ excision, the displacement had decreased to approximately $5 \mathrm{~mm}$, which was considered to be the result of osteoarthritis progression and other changes. ${ }^{20}$ However, no significant difference in the IE displacement was found during the 2-year observation period. ${ }^{20}$ One factor associated with the differences between the results of in vitro and in vivo studies is considered to be the effect of rotational stabilization by the patella; the patella is stabilized mediolaterally by the femoral trochlea. ${ }^{21}$ Therefore, the influence of the rotational instability induced by $\mathrm{CrCL}$ rupture on the walking cycle is considered to be relatively small. However, craniocaudal instability is present during every shift from the swing phase to the stance phase, and the presence of craniocaudal instability in the absence of the $\mathrm{CrCL}$ may greatly influence the progression of osteoarthritis. Hulse and colleagues ${ }^{22}$ reported that the extent of the injury to the articular cartilage after TPLO depends on the degree of CrCL damage. They evaluated the articular cartilage by arthroscopy after TPLO and found that when CrCL function was preserved, the articular cartilage was normal or nearly normal and that degeneration of the $\mathrm{CrCL}$ was less, whereas when the $\mathrm{CrCL}$ function was not preserved, the damage to the articular cartilage damage was worse. ${ }^{22}$ This factor may also be affected by the instability induced by TPLO that has been shown in the present study.

This study had several limitations. First, the influence of dynamic stabilizers could not be reproduced. Furthermore, clinical CrCL rupture in the canine stifle manifests as a chronic condition termed 'cranial cruciate ligament disease', which may involve a partial tear or complete rupture. ${ }^{23}$ Therefore, the severity of a CrCL injury depends on the amount of ligament degeneration. Cranial cruciate ligament degeneration, often associated with aging, can affect stifle instability. $^{24,25}$ Therefore, the age difference between the control and TPLO groups might be responsible for the differences in stability. A previous report showed that such degeneration occurs by the age of 5 years in dogs weighing more than $15 \mathrm{~kg}^{25}$ Hence, we considered the risk small because the dogs used in this study were $15 \mathrm{~kg}$ or less and were all under two and a half years of age. Cranial cruciate ligament rupture is also associated with the development of 
chronic periarticular fibrosis. ${ }^{23}$ The degenerative changes observed in clinical cases, such as osteoarthritis and periarticular fibrosis, are a compensatory response and progress when the $\mathrm{CrCL}$ is damaged. These secondary joint changes have the potential to compensate for instability with respect to IE and craniocaudal loads in stifles from TPLO-treated dogs. However, we could not imitate these compensatory mechanisms in the present study. Therefore, many clinical cases of ligament rupture caused by $\mathrm{CrCL}$ disease are expected to progress to stifle stabilization, which was not possible in the present study. Hence, this hypothesis must be tested in the future.

In conclusion, in the present study, TPLO provided effective craniocaudal stabilization during vertical compression in stifle joints with a transected $\mathrm{CrCL}$. However, it promoted instability under other test conditions following $\mathrm{CrCL}$ transection, which may be due to relative joint flexion inducing collateral ligament laxity. This instability might lead to more rapid progression of stifle osteoarthritis following TPLO. Therefore, TPLO should be performed while the $\mathrm{CrCL}$ is still partially functional to slow the progression of postoperative osteoarthritis.

\section{Authors' Contributions}

All authors contributed to conception of study, study design, acquisition of data and data analysis and interpretation. All authors also drafted, revised and approved the submitted manuscript.

\section{Conflict of Interest}

None declared.

\section{Acknowledgements}

We thank Dr Tatsuyuki Yoshida for assisting with the statistical analysis. We also thank Angela Morben, DVM, ELS, and Mark Cleasby, PhD, from Edanz Group (www. edanzediting.com/ac), for editing a draft of this manuscript.

\section{References}

1 Arnoczky SP, Marshall JL. The cruciate ligaments of the canine stifle: an anatomical and functional analysis. Am J Vet Res 1977; 38(11):1807-1814

2 Slocum B, Slocum TD. Tibial plateau leveling osteotomy for repair of cranial cruciate ligament rupture in the canine. Vet Clin North Am Small Anim Pract 1993;23(04):777-795

3 Krotscheck U, Nelson SA, Todhunter RJ, Stone M, Zhang Z. Long term functional outcome of tibial tuberosity advancement vs. tibial plateau leveling osteotomy and extracapsular repair in a heterogeneous population of dogs. Vet Surg 2016;45(02):261-268

4 Au KK, Gordon-Evans WJ, Dunning D, et al. Comparison of shortand long-term function and radiographic osteoarthrosis in dogs after postoperative physical rehabilitation and tibial plateau leveling osteotomy or lateral fabellar suture stabilization. Vet Surg 2010;39(02):173-180

5 Rayward RM, Thomson DG, Davies JV, Innes JF, Whitelock RG. Progression of osteoarthritis following TPLO surgery: a prospec- tive radiographic study of 40 dogs. J Small Anim Pract 2004;45 (02):92-97

6 Warzee CC, Dejardin LM, Arnoczky SP, Perry RL. Effect of tibial plateau leveling on cranial and caudal tibial thrusts in canine cranial cruciate-deficient stifles: an in vitro experimental study. Vet Surg 2001;30(03):278-286

7 Kanno N, Amimoto H, Hara Y, et al. In vitro evaluation of the relationship between the semitendinosus muscle and cranial cruciate ligament in canine cadavers. Am J Vet Res 2012;73 (05):672-680

8 Kim SE, Pozzi A, Banks SA, Conrad BP, Lewis DD. Effect of tibial plateau leveling osteotomy on femorotibial contact mechanics and stifle kinematics. Vet Surg 2009;38(01):23-32

9 Kanno N, Hara Y, Fukano S, et al. Tibial displacement with stifle joint flexion and cranial cruciate ligament transection in the dog. An ex vivo study using a robotic simulator. Vet Comp Orthop Traumatol 2014;27(04):277-284

10 Fujie H, Mabuchi K, Woo SL, Livesay GA, Arai S, Tsukamoto Y. The use of robotics technology to study human joint kinematics: a new methodology. J Biomech Eng 1993;115(03):211-217

11 Fujie H, Sekito T, Orita A. A novel robotic system for joint biomechanical tests: application to the human knee joint. J Biomech Eng 2004;126(01):54-61

12 Fujie H, Livesay GA, Fujita M, Woo SL. Forces and moments in sixDOF at the human knee joint: mathematical description for control. J Biomech 1996;29(12):1577-1585

13 Kimura K, Fujie H. Development of a novel robotic system for joint mechanical tests using a real-time controller. Jpn Soc Mech Eng 2015;81(826):14-00684(in Japanese)

14 Grood ES, Suntay WJ. A joint coordinate system for the clinical description of three-dimensional motions: application to the knee. J Biomech Eng 1983;105(02):136-144

15 Muir P. Physical examination of lame dogs. Compend Contin Educ Pract Vet 1997;19:1149-1161

16 Vasseur PB, Arnoczky SP. Collateral ligaments of the canine stifle joint: anatomic and functional analysis. Am J Vet Res 1981;42 (07):1133-1137

17 Knight RC, Thomson DG, Danielski A. Surgical management of pivot-shift phenomenon in a dog. J Am Vet Med Assoc 2017;250 (06):676-680

18 Slocum B, Devine T. Cranial tibial thrust: a primary force in the canine stifle. J Am Vet Med Assoc 1983;183(04):456-459

19 Boudrieau RJ. Tibial plateau leveling osteotomy or tibial tuberosity advancement? Vet Surg 2009;38(01):1-22

20 Tashman S, Anderst W, Kolowich P, Havstad S, Arnoczky S. Kinematics of the ACL-deficient canine knee during gait: serial changes over two years. J Orthop Res 2004;22(05):931-941

21 Flandry F, Hommel G. Normal anatomy and biomechanics of the knee. Sports Med Arthrosc Rev 2011;19(02):82-92

22 Hulse D, Beale B, Kerwin S. Second look arthroscopic findings after tibial plateau leveling osteotomy. Vet Surg 2010;39(03): 350-354

23 Hayashi K, Manley PA, Muir P. Cranial cruciate ligament pathophysiology in dogs with cruciate disease: a review. J Am Anim Hosp Assoc 2004;40(05):385-390

24 Döring AK, Junginger J, Hewicker-Trautwein M. Cruciate ligament degeneration and stifle joint synovitis in 56 dogs with intact cranial cruciate ligaments: correlation of histological findings and numbers and phenotypes of inflammatory cells with age, body weight and breed. Vet Immunol Immunopathol 2018;196:5-13

25 Vasseur PB, Pool RR, Arnoczky SP, Lau RE. Correlative biomechanical and histologic study of the cranial cruciate ligament in dogs. Am J Vet Res 1985;46(09):1842-1854 